https://helda.helsinki.fi

\title{
Systematic review of analgesics and dexamethasone for post-tonsillectomy pain in adults
}

Tolska, H. K.

2019-08

Tolska , H K, Hamunen, K, Takala , A \& Kontinen, V K 2019 , ' Systematic review of analgesics and dexamethasone for post-tonsillectomy pain in adults ' , British Journal of Anaesthesia , vol. 123 , no. 2 , pp. E397-E411 . https://doi.org/10.1016/j.bja.2019.04.063

http://hdl.handle.net/10138/306021

https://doi.org/10.1016/j.bja.2019.04.063

publishedVersion

Downloaded from Helda, University of Helsinki institutional repository.

This is an electronic reprint of the original article.

This reprint may differ from the original in pagination and typographic detail.

Please cite the original version. 


\title{
Systematic review of analgesics and dexamethasone for post- tonsillectomy pain in adults
}

\author{
H. K. Tolska ${ }^{1, *}$, K. Hamunen ${ }^{2}$, A. Takala ${ }^{1,2}$ and V. K. Kontinen ${ }^{1}$ \\ ${ }^{1}$ Division of Anesthesiology, Department of Anesthesiology, Intensive Care and Pain Medicine, University of Helsinki and \\ Helsinki University Hospital, Helsinki, Finland and ${ }^{2}$ National Supervisory Authority for Welfare and Health, Helsinki, \\ Finland
}

${ }^{*}$ Corresponding author. E-mail: kaisa.tolska@hus.fi

\begin{abstract}
Background: Intense pain can last several days after tonsillectomy. It is often undertreated and improved analgesic strategies that can be safely used at home are needed.

Methods: We conducted a systematic review and meta-analysis on the effectiveness of systemic medications used for post-tonsillectomy pain in adult and adolescent (13 yr old) patients. Studies were identified from PubMed, the Cochrane Library, and by hand searching reference lists from studies and review articles. Randomised, double-blind, placebocontrolled studies reporting on pain intensity or use of rescue analgesia were included.

Results: Twenty-nine randomised controlled trials representing 1816 subjects met the inclusion criteria. Follow-up time was $\leq 24 \mathrm{~h}$ in 15 studies, in which the majority were taking nonsteroidal anti-inflammatory drugs. Thirteen studies were suitable for meta-analysis. In pooled analysis, paracetamol, dexamethasone, and gabapentinoids reduced pain intensity on the day of operation. In individual studies, ketoprofen, ibuprofen, lornoxicam, parecoxib, rofecoxib, indomethacin and dextromethorphan reduced pain intensity, need for rescue analgesics, or both on the day of operation. Oral celecoxib for 2 postoperative weeks or i.v. ketamine on the day of operation were not effective at the studied doses. Dexamethasone in multiple doses provided analgesia beyond 1 postoperative day. Pain was moderate to strong in both study and control groups during the first postoperative week.

Conclusions: Single analgesics and dexamethasone provide only a weak to moderate effect for post-tonsillectomy pain on the day of operation and thus a multimodal analgesic strategy is recommended. Short follow-up times and clinical heterogeneity of studies limit the usefulness of results.
\end{abstract}

Keywords: adolescent; adult; analgesics; dexamethasone; multimodal analgesia; postoperative pain; tonsillectomy

Editor's key points

- The authors performed a systematic review and metaanalysis of pain management after tonsillectomy. Data were scarce and postoperative follow-up was short in the majority of studies.

- Paracetamol, NSAIDs, dexamethasone, gabapentinoids, and dextromethorphan showed weak to moderate analgesic effects on the day of operation.
- The authors suggest that multimodal analgesia is needed after tonsillectomy, and that further research on post-tonsillectomy pain is required, with follow-up over at least 1 week. 
Tonsillectomy is one of the most common procedures in ear, nose, and throat surgery. It is usually performed as a daystay procedure. ${ }^{1}$ Pain is the most common reason for physician contact after discharge, indicating that posttonsillectomy pain is intense and undertreated. ${ }^{2}$ Patients need analgesics that are effective and can be used safely at home.

Adults have a different pattern of pain compared with children; this is related to the different indications and techniques of tonsillectomy. ${ }^{3,4}$ In adults, the surgery is usually performed for chronic infection with scarred tonsils that requires dissection with coagulation, thus causing intense and longer-lasting pain. ${ }^{5}$ In children, the indication is usually hypertrophy or recurrent acute infections with smaller changes in tonsil tissues than in adults. ${ }^{6}$

Multimodal analgesia has become the standard of care in postoperative pain management. Combinations of analgesics with different sites or modes of action are commonly used to improve analgesia and to reduce the doses of individual analgesics (especially opioids) and to reduce adverse effects. Adverse effects of opioids, such as sedation and respiratory depression, are particularly dangerous when the surgery has been performed along the respiratory tract. The benefits of analgesics and dexamethasone used for post-tonsillectomy pain require review. Systematic reviews on various analgesics for post-tonsillectomy pain have been published for paediatric and adolescent patients. ${ }^{7}$ Paracetamol (alone and in combination with ibuprofen) for acute postoperative pain has been analysed in systematic reviews that have included some tonsillectomy studies. ${ }^{8-10}$ Also, the effects of NSAIDs on the risk for post-tonsillectomy haemorrhage (PTH) have been studied widely. ${ }^{11-13}$ Reviews on the effect of dexamethasone on post-tonsillectomy morbidity have mainly focused on the risk for PTH and postoperative nausea and vomiting (PONV); we found one review that included pain as a clinical endpoint in adults. ${ }^{14}$ Additionally, one study reviewed the effect of dexamethasone for post-tonsillectomy pain only. ${ }^{15}$ Reviews on gabapentinoids for acute pain after general surgery, posttonsillectomy, and after head-neck surgery have been published. ${ }^{16-18}$ However, the effect of various analgesics on posttonsillectomy pain in adults has not been systemically reviewed, to our knowledge.

We performed a systematic review and meta-analysis of published studies to assess the effect of systemic analgesics in the treatment of post-tonsillectomy pain in adult and adolescent patients. Randomised, double-blind, placebocontrolled studies reporting at least one analgesic outcome (such as incidence or intensity of pain) or use of rescue analgesics were included.

\section{Methods}

The Preferred Reporting Items for Systematic Reviews and Meta-Analyses (PRISMA) statement guidelines were followed in performing and reporting the review. ${ }^{19}$

\section{Search strategy}

The following electronic databases were searched to identify published or ongoing RCTs: Pubmed, Ovid MEDLINE, The Cochrane Central Register of Controlled Trials (CENTRAL), and Cochrane Database of Systematic Reviews (CDSR). We used the following search terms: (tonsillectomy or tonsillectomy* or tonsillotomy or post tonsillectomy)
AND (pain or pain* or analgesia or analgesic* or narcotics). The search retrieved only a few studies on dexamethasone. Therefore, targeted searches were made using the following terms: (tonsillectomy or tonsillectomy* or tonsillotomy or post tonsillectomy) AND (dexamethasone* or corticosteroids*). We searched trial registries www.clinicaltrials.gov and www.eudraCT.com for unpublished trials. We handsearched reference lists of included studies and identified reviews for additional potential trials. The search was performed in February 2017 and conducted by one author (KT). Three reviewers (KT, AT, KH) independently screened all studies for eligibility by titles and abstracts, and a decision was made on whether to obtain full-text publications. These studies were then inspected for relevance and a decision was made on whether to include or exclude them. Disagreements between reviewers were resolved by consensus.

\section{Inclusion criteria}

Types of studies: double-blind placebo-controlled randomised studies (RCTs) of systemic analgesics for post-tonsillectomy pain in adults or adolescents. Only publications in English were included.$^{20}$ Studies published before 1980 were excluded, as operation techniques have evolved and hence the study settings would not be comparable with those in more recent years.

Types of participants: adults and adolescents ( $\geq 13$ yr old) undergoing tonsillectomy for any indication as inpatient or outpatient. Studies on paediatric patients and mixed studies of adult and paediatric patients were excluded. Studies of less than 10 participants in a group were excluded.

Types of intervention: administration of systemic (oral, rectal, i.m., or i.v.) analgesics (paracetamol, NSAIDs, gabapentin, pregabalin, dextromethorphan, ketamine) and dexamethasone for prevention or treatment of pain. Studies on topical, infiltration, and regional analgesics were excluded.

Types of outcome measures: the primary outcome was incidence or intensity of pain, or both; secondary outcomes were the use of rescue analgesia and adverse effects. Studies that did not report at least one analgesic outcome measure were excluded.

\section{Data collection}

We developed a data abstraction table, pilot-tested it on five randomly selected studies, and refined it accordingly. Data collection from included studies was performed by one author (KT) and reviewed by two other authors (AT, KH). Disagreements were resolved by discussion between three authors (HKT, AT, KH). A fourth author (VKK) was consulted if no agreement was reached.

The following data on study participants were extracted: age, number of patients, ASA status (ASA Physical Status Classification System), number and reason for dropouts, type and technique of operation, type of anaesthesia (local or general, whether analgesics were used during anaesthesia), type of intervention (type, dose, frequency, and timing of analgesics [preoperative, intraoperative, or postoperative]), additional and rescue analgesics, method of pain measurement and by whom (patient or observer), type of comparator (placebo), type of outcome measure (pain intensity before and after medication, rescue analgesics, 
return to daily activities, adverse effects), length of followup, and study design (randomisation, blinding of randomisation, and intervention).

\section{Quality assessment and risk of bias in and across individual studies}

Three authors (HKT, KH, AT) independently assessed the validity and potential bias of included studies by evaluating the adequacy of randomisation and concealment of allocation (sequence generation), blinding of patients, healthcare providers, data collectors, and outcome assessors, intention-totreat, and for incomplete outcome data and selective outcome reporting. The Cochrane risk of bias tool was used for assessment of methodological quality of included studies. ${ }^{21}$ Publication bias of included studies was determined by a funnel plot.

\section{Statistical analysis and synthesis}

Data were analysed by calculating the mean difference with the corresponding 95\% confidence intervals (CI). We performed meta-analyses of included studies when a group of studies in which clinical heterogeneity was sufficiently small could be identified. Data were analysed using Review Manager (RevMan; version 5.3; Copenhagen: The Nordic Cochrane Centre, The Cochrane Collaboration, 2014). Statistical heterogeneity was analysed with a $\chi^{2}$ test and $\mathrm{I}^{2}$ was calculated. Pooled meta-analyses were not performed in case clinical heterogeneity was too great or reporting of outcome measures varied excessively within a group of analgesics. Instead, narrative synthesis was used to compare studies qualitatively by using significant differences reported in original studies. In the overall interpretation of the results, we consider that 'no more than mild pain' (less than $3 / 10$ in the pain scale) is an acceptable result in clinical practice (moderate pain $3-6 / 10$, strong/severe pain 6-10/10). ${ }^{22}$

\section{Results}

We retrieved 2219 citations from an electronic database search and an additional 14 citations from reference lists of reviews (Fig. 1). After excluding duplicates and articles that did not fulfil the inclusion criteria, full-text articles of 81 studies were assessed for eligibility, based on the title and abstract. Of these, 29 studies representing 1816 patients met the inclusion criteria, of which 13 were suitable for pooled meta-analysis.

\section{Characteristics of the included studies}

The main characteristics and results of the included studies are shown in Table 1. A detailed description is shown in Supplementary Appendix S1. Two studies investigated paracetamol, ${ }^{23,24}$ nine NSAIDs, ${ }^{25-33} 10$ dexamethasone, ${ }^{34-43}$ three gabapentin, ${ }^{44-46}$ one pregabalin, ${ }^{47}$ two dextromethorphan, ${ }^{48,49}$ one ketamine, ${ }^{50}$ and one oxycodone. ${ }^{51}$ Patients undergoing adenotonsillectomy were included in two studies. ${ }^{28,33}$ In the remaining studies, all patients underwent tonsillectomy. Surgery was performed under general anaesthesia in 27 studies; in two studies the type of anaesthesia was not reported. ${ }^{25,41}$ The surgical method was described in 19 studies.

Patients in one study received dexamethasone in addition to study medication. ${ }^{33}$ Additional preoperative or postoperative analgesics were given in 13 studies, no additional analgesics were given in 10 studies, and information was not provided in six studies (Table 1). Rescue analgesics were given in 25 studies, were not allowed in one study, ${ }^{37}$ and information was not provided in three studies. ${ }^{34,43,51}$

Study medications were administered before operation in eight studies, , $^{29-31,45-49}$ before operation and intraoperatively in one study, ${ }^{44}$ intraoperatively in seven studies, $8,26,34,35,38,43,50$ intraoperatively and after operation in five studies, $27,32,36,37,39$ after operation in seven studies, , $^{23-25,33,40,41,51}$ and before and after operation in one study. ${ }^{44}$ One study compared intraoperative and postoperative administration of the study medication with placebo. ${ }^{28}$ Another study compared preoperative, intraoperative, and postoperative administration of the study medication with placebo. ${ }^{42}$ There were 17 singledose and 12 multiple-dose studies. In studies that compared more than one analgesic with placebo, only one study arm was analysed. The duration of follow-up times ranged from $70 \mathrm{~min}$ to 10 days (Table 2). If 4-h or 24-h data were not available, we used the datapoint closest to time. There were three multicentre studies. $^{32,39,44}$

Pain intensity was the primary outcome in 11 studies $^{29-33,35,36,38,39,44,47}$ and rescue analgesia in six studies (number of doses during follow-up, ${ }^{23}$ proportion of patients requiring rescue analgesics to maintain visual analogue scale [VAS] at rest [VASr] $\leq 30 \mathrm{~mm}$ and VAS on swallowing (VASs) $\leq 50 \mathrm{~mm},{ }^{24}$ total dose of rescue analgesics, ${ }^{27,50}$ proportion of patients that needed rescue analgesics, ${ }^{28}$ and time to first analgesic request after discharge from operating room ${ }^{49}$ ). Twelve studies did not specify the primary outcome but reported data on pain intensity, ${ }^{34,37,40-43,45,46,48,51}$ pain relief, ${ }^{25}$ and rescue analgesia. ${ }^{26}$ We converted pain intensity values to a $0-10$ scale when a $0-5$ scale was used in a study. Pain intensity scales of $0-6$ and $0-4$ were used in one dexamethasone study ${ }^{42}$ and in one gabapentin study, ${ }^{44}$ respectively; in these studies, we considered that converting values to $0-10$ scales would not be appropriate, and they were excluded from pain intensity comparisons in meta-analyses.

Pain intensity was reported in all but one study ${ }^{26}$ in which the only need for rescue analgesics was evaluated (total number of doses and time to first rescue analgesic). Pain intensity was reported by the patient in 11 studies and by an observer in 10 studies. Seven studies did not report who assessed pain intensity. The criteria for administration of rescue analgesia were reported in 24 studies, of which 10 studies reported a specified pain intensity value for administration of rescue analgesia.

\section{Quality and risk of bias of included studies}

All included studies were randomised, double-blind, and placebo controlled. The risk of bias graph is presented in Fig. 2 and the risk of bias summary in Supplementary Appendix S2. Detailed characteristics of bias in included studies are presented in Supplementary Appendix S3.

\section{Pain intensity after tonsillectomy}

Tonsillectomy caused moderate to severe pain that lasted for several days (Fig. 3). During the first postoperative week, pain intensities (median or mean as shown in figures) in both placebo and study groups ranged from 4 to 8 (pain intensity scale 0-10). By the end of the first postoperative week (7 POD), pain intensity had decreased to less than $4 / 10$ in the majority of study groups, whereas in the majority of placebo groups, pain 


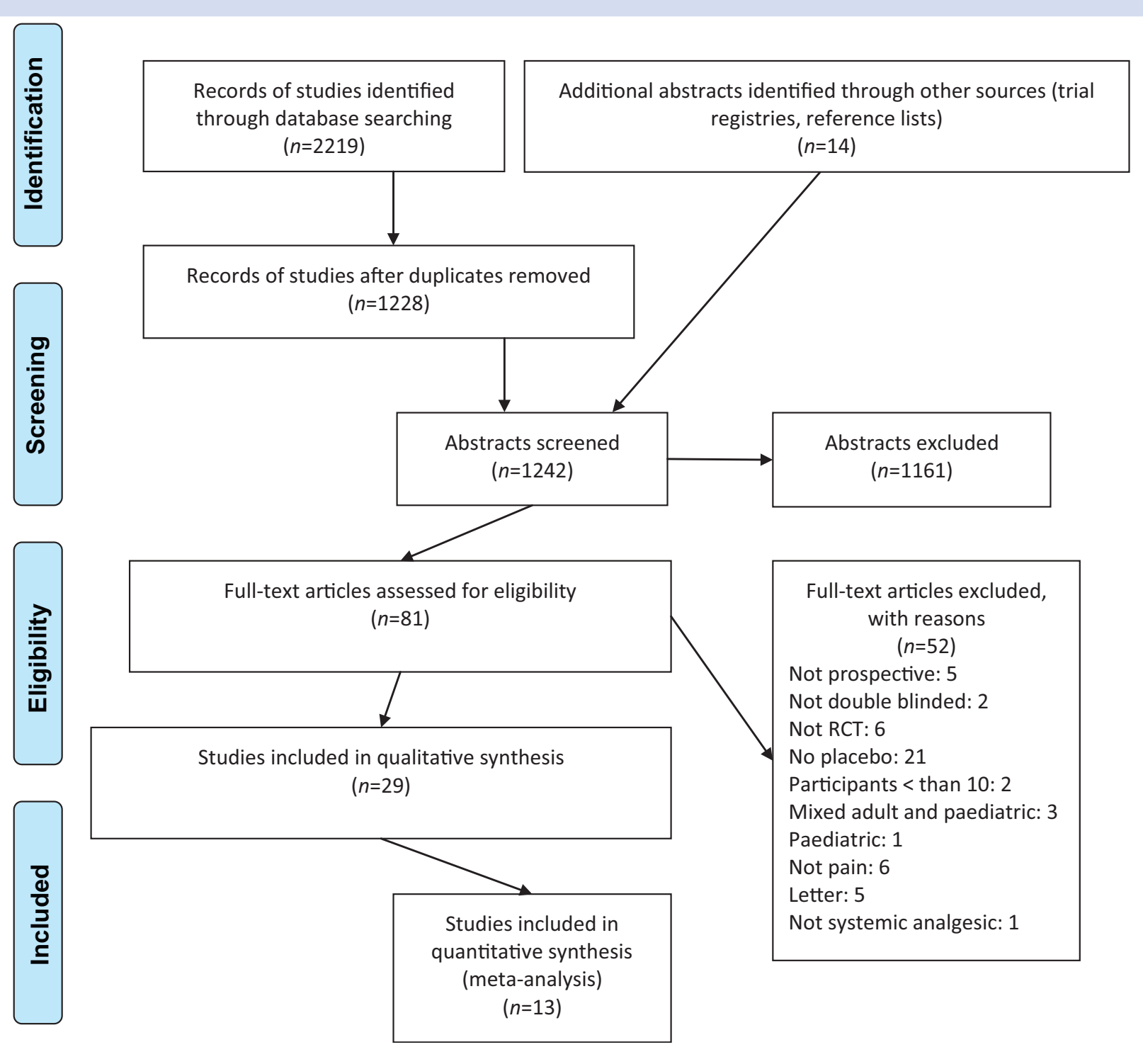

Fig 1. Preferred Reporting Items for Systematic Reviews and Meta-Analyses (PRISMA) flow diagram.

intensity was still greater than $4 / 10$ (Fig. $3 b$ and a, respectively).

Randomised studies comparing paracetamol with placebo

Two studies involving 153 participants ${ }^{23,24}$ are summarised in Table 1 (Supplementary Appendix S1). The pooled estimate for intraoperatively administered paracetamol 1-2 g i.v. showed a statistically significant decrease in pain intensity (VASs) at $4 \mathrm{~h}$ ( -0.88 [95\% CI -1.66 to -0.09$], P=0.03)$, equivalent to an $18 \%$ reduction compared with the control group. Both studies (single dose of $2 \mathrm{~g}$ i.v. intraoperatively ${ }^{24}$ and multiple doses up to $4 \mathrm{~g}$ i.v. on the day of operation ${ }^{23}$ ) reported a decreased need for opioids within $24 \mathrm{~h}$. One study ${ }^{24}$ that reported incidence of
PTH found no association between paracetamol and increased risk for $\mathrm{PTH}$.

Randomised studies comparing NSAIDs with placebo Nine studies involving 638 participants ${ }^{25-33}$ are summarised in Table 1 (Supplementary Appendix S1). Only two studies ${ }^{27,28}$ reported pain intensity values as mean with standard deviation (SD) or standard error of mean (SEM was converted to SD) for which a pooled meta-analysis was possible. Intraoperative ketoprofen failed to decrease pain intensity at $2 \mathrm{~h}$ and at $24 \mathrm{~h}$ (VASs scale $0-10)(-0.82[95 \%$ CI -2.10 to 0.45$], P=0.21$ and -0.67 [95\% CI -1.53 to 0.19 ], $P=0.13$, respectively) (Fig. 4). Both studies reported a reduced need for rescue analgesics within $24 \mathrm{~h}$. 
Table 1 Main characteristics and results of the included studies. intraop., intraoperatively; max, maximal; $n$, number; No., number; NS, not significant; POD, postoperative day; postop., after operation; preop., before operation; SD, standard deviation; sEMG, surface electromyography; TCI, target controlled infusion; VAS, visual analogue scale; VASr, VAS at rest; VASs, VAS on swallowing; VRS, verbal rating scale

\begin{tabular}{|c|c|c|c|c|c|}
\hline $\begin{array}{l}\text { Reference (and } \\
\text { year) }\end{array}$ & $\begin{array}{l}\text { No. of patients, } \\
\text { active/placebo }\end{array}$ & $\begin{array}{l}\text { Study analgesics } \\
\text { Study arm } \\
\text { Placebo } \\
\text { Time of administration }\end{array}$ & $\begin{array}{l}\text { Rescue analgesic, time of } \\
\text { administration }\end{array}$ & $\begin{array}{l}\text { Duration of } \\
\text { follow-up } \\
\text { (days) }\end{array}$ & Analgesic outcome results (study drug us placebo) \\
\hline $\begin{array}{l}\text { Atef and } \\
\text { Fawaz }^{23}(2008)\end{array}$ & $38 / 38$ & $\begin{array}{l}\text { Paracetamol } 1 \mathrm{~g} \text { i.v. saline i.v. } \\
\text { Intraop., } 6,12,18 \mathrm{~h}\end{array}$ & $\begin{array}{l}\text { Pethidine } 1 \mathrm{mg} \mathrm{kg}^{-1} \text { i.m. if } \\
\text { VASr }>30,0-24 \mathrm{~h}\end{array}$ & 1 & $\begin{array}{l}\text { Paracetamol reduced total dose of i.m. pethidine during } 0-24 \mathrm{~h} \\
(P<0.001), n \text { of pethidine doses/patient } 0-24 \mathrm{~h}(P<0.001) \text { and } \% \\
\text { of patients that needed pethidine }(P<0.05) \\
\text { Paracetamol decreased VASs and VASr at } 2 \text { and } 3 \mathrm{~h}(P<0.05)\end{array}$ \\
\hline $\begin{array}{l}\text { Salonen and } \\
\text { colleagues } \\
\text { (2009) }\end{array}$ & $39 / 37 / 38$ & $\begin{array}{l}\text { Paracetamol } 2 \text { g i.v. } \\
\text { Paracetamol } 1 \text { g i.v. } \\
\text { Saline i.v. } \\
10 \text { min postop. }\end{array}$ & $\begin{array}{l}\text { Oxycodone } 2 \mathrm{mg} \text { i.v. if } \\
\text { VASr }>30 \text { or VASs }>50,0 \\
-6 \mathrm{~h}\end{array}$ & 6 & $\begin{array}{l}\text { Proportion of patients that needed oxycodone } 0-6 \mathrm{~h} \text { NS } \\
\text { Paracetamol } 2 \mathrm{~g} \text { reduced } n \text { of oxycodone doses/patient } 0-6 \mathrm{~h} \\
(P=0.002) \text {, paracetamol } 1 \mathrm{~g} \text { NS. Time to } 1 \text { st dose of oxycodone } \\
\text { NS. Pain intensity NS }\end{array}$ \\
\hline $\begin{array}{l}\text { Parker and } \\
\text { colleagues } \\
\text { (1986) }\end{array}$ & $44 / 33 / 33$ & $\begin{array}{l}\text { Ibuprofen } 600 \mathrm{mg} \text { p.o. } \\
\text { Acetylsalicylic acid } 600 \mathrm{mg} \text { p.o. } \\
30 \mathrm{ml} \text { syrup p.o. } \\
0-4 \text { times daily for } 0-6 \text { postop. } \\
\text { days as needed }\end{array}$ & $\begin{array}{l}\text { Analgesia p.o. or i.m., dose } \\
\text { or type unclear, hospital } \\
\text { Paracetamol, home }\end{array}$ & 6 & $\begin{array}{l}\text { Ibuprofen provided pain relief at } 30 \mathrm{~min} \text { (\% of patients with pain } \\
50 \% \text { gone) }(P<0.05) \text {, at } 4 \mathrm{~h} \text { ibuprofen and acetylsalicylic acid NS } \\
\text { Analgesic consumption: ibuprofen and acetylsalicylic acid } 3-4 \\
\text { daily, placebo less, no numerical data. Rescue analgesia: no } \\
\text { one requested }\end{array}$ \\
\hline $\begin{array}{l}\text { Rorarius and } \\
\text { colleagues } \\
\text { (1993) }\end{array}$ & $21 / 21 / 21$ & $\begin{array}{l}\text { Indomethacin } 50 \mathrm{mg} \text { i.v. } \\
\text { Diclofenac } 75 \mathrm{mg} \text { i.v. } \\
\text { Saline i.v. } \\
\text { Intraop. }\end{array}$ & $\begin{array}{l}\text { Oxycodone } 3 \text { mg i.v., 0-70 } \\
\text { min }\end{array}$ & $<1$ & $\begin{array}{l}\text { Indometacin reduced } n \text { of oxycodone doses/group }(P=0.05) \text {. } \\
\text { Time to 1st rescue analgesic NS. Proportion of patients that } \\
\text { needed oxycodone NS }\end{array}$ \\
\hline $\begin{array}{l}\text { Tarkkila and } \\
\text { Saarnivaara }{ }^{27} \\
\text { (1999) }\end{array}$ & $20 / 20 / 20 / 20$ & $\begin{array}{l}\text { Ketoprofen } 100 \mathrm{mg} \text { i.v. } \\
\text { Diclofenac } 75 \mathrm{mg} \text { i.v. } \\
\text { Ketorolac } 30 \mathrm{mg} \text { i.v. } \\
\text { Saline i.v. } \\
\text { Intraop., } 6,12 \mathrm{~h}\end{array}$ & $\begin{array}{l}\text { Oxycodone } 0.05 \mathrm{mg} \mathrm{kg}^{-1} \\
\text { i.v., } 0-2 \mathrm{~h} \\
\text { Oxycodone } 0.1 \mathrm{mg} \mathrm{kg}^{-1} \\
\text { i.m., } 2-24 \mathrm{~h}\end{array}$ & 1 & $\begin{array}{l}\text { All NSAIDs reduced total } n \text { of oxycodone doses/patient } 0-24 \mathrm{~h} \\
(P<0.05) \\
\text { Ketorolac reduced proportion of patients that requested } \\
\text { oxycodone }(P<0.05) \text {. Pain intensity NS }\end{array}$ \\
\hline $\begin{array}{l}\text { Salonen and } \\
\text { colleagues } \\
\text { (2001) }\end{array}$ & $41 / 40 / 25$ & $\begin{array}{l}\text { Ketoprofen } 0.5 \mathrm{mg} \mathrm{kg}^{-1} \text { i.v. bolus } \\
\text { with ketoprofen infusion } 3 \mathrm{mg} \\
\mathrm{kg}^{-1} \\
\text { Saline i.v. bolus with infusion } \\
\text { Bolus at induction ('intra') or in } \\
\text { PACU ('post') with } 24 \mathrm{~h} \text { infusion } \\
\text { in both }\end{array}$ & $\begin{array}{l}\text { Oxycodone } 0.05 \mathrm{mg} \mathrm{kg}^{-1} \\
\text { i.v. if VASr } \geq 30,0-4 \mathrm{~h} \\
\text { Oxycodone } 0.1 \mathrm{mg} \mathrm{kg}^{-1} \\
\text { i.m. if } \mathrm{VASr} \geq 30,4-24 \mathrm{~h}\end{array}$ & 1 & $\begin{array}{l}\text { Proportion of patients that needed oxycodone in } P A C U \text { } 0-4 \mathrm{~h} \text { NS, } \\
\text { intra- and post-ketoprofen at ward } 5-24 \mathrm{~h} \text { reduced }(P=0.002), 0 \\
-24 \mathrm{~h} \text { NS } \\
\text { Intra- and post-ketoprofen reduced } n \text { of oxycodone doses/ } \\
\text { patient at all time intervals us placebo: } 0-4 \mathrm{~h}(P=0.03, P=0.04 \text {, } \\
\text { respectively), } 5-24 \mathrm{~h}(P<0.01, P<0.01 \text {, respectively) and } 0-24 \mathrm{~h} \\
(P<0.01, P<0.01 \text { respectively). Total dose of oxycodone NS }\end{array}$ \\
\hline $\begin{array}{l}\text { Naesh and } \\
\text { colleagues }{ }^{29} \\
(2005)\end{array}$ & $20 / 20$ & $\begin{array}{l}\text { Rofecoxib } 50 \text { mg p.o. identical } \\
\text { capsules p.o. } \\
1.5 \text { h preop. }\end{array}$ & $\begin{array}{l}\text { Morphine } 2.5 \mathrm{mg} \text { i.v. until } \\
\text { VAS }<3 \text { or patient } \\
\text { comfortable, } 0-24 \mathrm{~h}\end{array}$ & 1 & $\begin{array}{l}\text { VAS } 0-24 \mathrm{~h} \text { NS. Rofecoxib reduced strong pain }(\mathrm{VAS}>5) 0-8 \mathrm{~h} \text { : } \\
(P=0.02) \\
\text { Rofecoxib reduced total dose of morphine during } 0-8 \mathrm{~h} \\
(P<0.04) \\
\text { Time to } 1 \text { st rescue dose NS }\end{array}$ \\
\hline $\begin{array}{l}\text { Ismail and } \\
\text { Mowafi } \\
(2010)\end{array}$ & 20/19 & $\begin{array}{l}\text { Lornoxicam } 16 \mathrm{mg} \text { i.v. } \\
\text { Saline i.v. } \\
30 \text { min before induction }\end{array}$ & $\begin{array}{l}\text { Solpadeine plus } \\
\text { (paracetamol, codeine, } \\
\text { and caffeine in ratio of } \\
\text { 500:8:30 mg, } \\
\text { respectively) } \\
2 \text { Tablets p.o. every } 4 \mathrm{~h} \\
\text { and Tramal } 50 \mathrm{mg} \text { i.v. if } \\
\text { needed, } 0-24 \mathrm{~h}\end{array}$ & 1 & $\begin{array}{l}\text { Lornoxicam reduced VASs and VASr at } 4 \mathrm{~h}(P<0.05) \text { and VASs at } \\
24 \mathrm{~h}(P<0.05) \\
\text { Lornoxicam increased time to } 1 \text { st dose of paracetamol }(P<0.05) \\
\text { Lornoxicam reduced total dose of paracetamol (in Solpadeine) } \\
0-12 \mathrm{~h}(P<0.05) \text { and } 0-24 \mathrm{~h}(P<0.05) \text {. None needed Tramadol }\end{array}$ \\
\hline
\end{tabular}




\begin{tabular}{|c|c|c|c|c|c|}
\hline $\begin{array}{l}\text { Reference (and } \\
\text { year) }\end{array}$ & $\begin{array}{l}\text { No. of patients, } \\
\text { active/placebo }\end{array}$ & $\begin{array}{l}\text { Study analgesics } \\
\text { Study arm } \\
\text { Placebo } \\
\text { Time of administration }\end{array}$ & $\begin{array}{l}\text { Rescue analgesic, time of } \\
\text { administration }\end{array}$ & $\begin{array}{l}\text { Duration of } \\
\text { follow-up } \\
\text { (days) }\end{array}$ & Analgesic outcome results (study drug us placebo) \\
\hline $\begin{array}{l}\text { Mowafi and } \\
\text { colleagues } \\
(2011)\end{array}$ & $20 / 20$ & $\begin{array}{l}\text { Lornoxicam } 16 \mathrm{mg} \text { i.v. } \\
\text { Saline i.v. } \\
30 \text { min before induction }\end{array}$ & $\begin{array}{l}\text { Diclofenac } 50 \text { mg p.r., } 0.5 \\
-24 \text { h }\end{array}$ & 1 & $\begin{array}{l}\text { Lornoxicam reduced VRSmax }(P<0.001) \text { and VRSr at } 4 \text { and } 24 \mathrm{~h} \\
(P<0.001) \text { and VRSs at } 4 \mathrm{~h}(P<0.03) \text {, at } 24 \mathrm{~h} \text { NS } \\
\text { Lornoxicam reduced total dose and increased time to 1st dose } \\
\text { of diclofenac }(P<0.001, P<0.001)\end{array}$ \\
\hline $\begin{array}{l}\text { Xie and } \\
\text { colleagues } \\
(2012)\end{array}$ & $40 / 40$ & $\begin{array}{l}\text { Parecoxib } 40 \mathrm{mg} \text { i.v. } \\
\text { Saline i.v. } \\
\text { Intraop.,10 h }\end{array}$ & $\begin{array}{l}\text { PACU: fentanyl } 0.2 \mu \mathrm{gg}^{-1} \\
\text { i.v. at } 10 \text { min intervals if } \\
\text { VRS } \geq 5 \text { (max } 0.1 \mathrm{mg} \text { ) } \\
\text { Ward: paracetamol } 0.5 \mathrm{~g} \\
\text { i.v. min. } 6 \text { h intervals } \\
\text { (max } 2 \text { g daily) }\end{array}$ & 2 & $\begin{array}{l}\text { Parecoxib reduced VRSs at } 2 \mathrm{~h}(P<0.001) \text {, and VRSs and VRSr at all } \\
\text { time points }(P<0.001) \\
\text { Parecoxib reduced proportion of patients that needed } \\
\text { paracetamol at ward }(P<0.05) \text {, fentanyl at PACU NS }\end{array}$ \\
\hline $\begin{array}{l}\text { Ng and } \\
\text { colleagues }{ }^{33}( \\
\text { 2017) }\end{array}$ & $40 / 40$ & $\begin{array}{l}\text { Celecoxib } 200 \mathrm{mg} \times 2 \text { p.o. identical } \\
\text { placebo capsules p.o. } \\
1-10 \text { POD }\end{array}$ & $\begin{array}{l}\text { PACU: morphine i.v. or } \\
\text { oxycodone } 5 \mathrm{mg} \text { p.o. } \\
\text { every } 6 \text { h or when } \\
\text { needed } \\
\text { Home: oxycodone } 5 \mathrm{mg} \\
\text { p.o., max } 100 \mathrm{mg} \text { daily }\end{array}$ & 10 & $\begin{array}{l}\text { Pain intensity (overall and daily) NS. } \\
\text { Total dose of oxycodone NS. } \\
\text { 1st pain-free day and return to normal activities NS }\end{array}$ \\
\hline $\begin{array}{l}\text { Fields and } \\
\text { colleagues }^{34} \\
\text { (1994) }\end{array}$ & $29 / 29$ & $\begin{array}{l}\text { Dexamethasone } 8 \mathrm{mg} \text { i.v. } \\
\text { Saline i.v. } \\
\text { Intraop. }\end{array}$ & $\begin{array}{l}\text { Elixir: paracetamol } 250 \mathrm{mg} \\
\text { with codeine } 7.5 \mathrm{mg} \text { in } 5 \\
\mathrm{ml} \text { p.o., } 1-7 \mathrm{POD}\end{array}$ & 7 & $\begin{array}{l}\text { Dexamethasone decreased pain intensity at } 4 \mathrm{~h}(\mathrm{P}<0.05) \text {, from } 10 \\
\text { pm to } 7 \text { th POD NS } \\
\text { Total and daily dose of paracetamol+codeine elixir } 1-10 \text { POD } \\
\text { NS }\end{array}$ \\
\hline $\begin{array}{l}\text { Carr and } \\
\text { colleagues } \\
\text { (1999) }\end{array}$ & $15 / 14$ & $\begin{array}{l}\text { Dexamethasone } 20 \mathrm{mg} \text { i.v. } \\
\text { Saline i.v. } \\
\text { Intraop. }\end{array}$ & $\begin{array}{l}\text { PACU: morphine i.v., } \\
\text { pethidine i.v. } \\
\text { Ward: codeine and } \\
\text { paracetamol p.o. } \\
\text { Home: codeine p.o. every } \\
4 \text { h or if needed and } \\
\text { paracetamol, 1-10 POD } \\
\text { (doses unclear) }\end{array}$ & 10 & $\begin{array}{l}\text { Pain intensity ( }>2 \text { VAS change) NS. N of rescue analgesic dose in } \\
\text { PACU NS } \\
\text { Dexamethasone reduced total dose of pethidine/patient } \\
\text { ( } P=0.03) \text {, but not total dose of morphine/patient. Proportion of } \\
\text { patients that needed rescue analgesics in PACU NS. Daily } \\
\text { doses of codeine and paracetamol 1-10 POD NS. Number of } \\
\text { days taken off from school or work and time until tolerating } \\
\text { normal diet NS }\end{array}$ \\
\hline $\begin{array}{l}\text { Stewart and } \\
\text { colleagues } \\
(2002)\end{array}$ & $48 / 52$ & $\begin{array}{l}\text { Dexamethasone } 8 \mathrm{mg} \text { i.v. at } \\
\text { induction, } 2 \text { mg p.o. at } 10 \mathrm{pm}, 2 \\
\text { mg p.o. twice daily for } 1-4 \text { POD, } \\
\text { then } 2 \text { mg daily for } 5-8 \text { POD } \\
\text { Saline i.v. and placebo tablets } \\
\text { p.o. } \\
\text { Intraop. and postop. }\end{array}$ & $\begin{array}{l}\text { PACU: morphine } 2 \mathrm{mg} \text { i.v. } \\
\text { Home: Co-codamol } \\
\text { (paracetamol } 1 \mathrm{~g} \text { with } \\
\text { codeine } 16 \mathrm{mg} \text { ) } 2 \text { tablets } \\
\text { p.o. every } 6 \mathrm{~h} \text { or if } \\
\text { needed, } 1-9 \text { POD }\end{array}$ & 9 & $\begin{array}{l}\text { Dexamethasone reduced pain intensity on all } 1-9 \text { POD, except } \\
\text { on } 0 \text { and } 2 \text { nd POD, } P<0.05 \text {. Dexamethasone decreased } \\
\text { proportion of patients that needed rescue analgesics (Co- } \\
\text { codamol }>8 \text { per day) }(P=0.024) \text {, and total dose of Co-codamol/ } \\
\text { day on } 2-7 \text { POD }(P<0.05) \text {. Total dose of morphine in PACU } \\
\text { unclear }\end{array}$ \\
\hline $\begin{array}{l}\text { Al-Shehri }{ }^{37} \\
(2004)\end{array}$ & $15 / 15$ & $\begin{array}{l}\text { Dexamethasone } 6 \text { mg i.v. } \\
\text { Saline i.v. } \\
\text { Intraop.,8, } 16 \mathrm{~h}\end{array}$ & None & 10 & $\begin{array}{l}\text { Dexamethasone reduced VAS on } 1 \text { POD }(P<0.05) \text { and on } 8 \text { POD } \\
(P<0.05) \text {. Total dose of analgesics NS. Patients in } \\
\text { dexamethasone group reduced their analgesic intake earlier } \\
\text { during } 2 \text { nd week; at } 7,8 \text {, and } 10 \text { POD, } P \text { unclear }\end{array}$ \\
\hline $\begin{array}{l}\text { Rujirojindakul } \\
\text { and } \\
\text { colleagues }^{38} \\
(2008)\end{array}$ & $25 / 25$ & $\begin{array}{l}\text { Dexamethasone } 20 \mathrm{mg} \text { i.v. } \\
\text { Saline i.v. } \\
\text { Intraop. }\end{array}$ & $\begin{array}{l}\text { PACU: fentanyl i.v. every } 10 \\
\text { min if VRS }>5 \text { (dose } \\
\text { unclear) } \\
\text { Ward: paracetamol } 10 \\
\text { mg kg }{ }^{-1} \text { p.o. every } 6 \mathrm{~h} \text { if } \\
\text { VRS }>5 \text { or morphine } 3 \mathrm{mg} \\
\text { i.v. every } 1 \mathrm{~h} \text { if VRS }>5\end{array}$ & 2 & $\begin{array}{l}\text { Dexamethasone reduced VRS at } 4 \mathrm{~h}(P=0.03) \text {, other times NS } \\
\text { Time to first rescue analgesic NS. Total dose of rescue } \\
\text { analgesics } 0-48 \mathrm{~h} \text { NS }\end{array}$ \\
\hline
\end{tabular}




\begin{tabular}{|c|c|c|c|c|c|}
\hline $\begin{array}{l}\text { Reference (and } \\
\text { year) }\end{array}$ & $\begin{array}{l}\text { No. of patients, } \\
\text { active/placebo }\end{array}$ & $\begin{array}{l}\text { Study analgesics } \\
\text { Study arm } \\
\text { Placebo } \\
\text { Time of administration }\end{array}$ & $\begin{array}{l}\text { Rescue analgesic, time of } \\
\text { administration }\end{array}$ & $\begin{array}{l}\text { Duration of } \\
\text { follow-up } \\
\text { (days) }\end{array}$ & Analgesic outcome results (study drug us placebo) \\
\hline $\begin{array}{l}\text { Lachance and } \\
\text { colleagues } \\
\text { (2008) }\end{array}$ & $37 / 49$ & $\begin{array}{l}\text { Dexamethasone } 8 \mathrm{mg} \text { i.v. and } 8 \\
\text { mg p.o. on } 0 \text { POD at home, } 6 \mathrm{mg} \\
\text { twice on } 1 \text { POD, } 4 \text { mg twice on } 2 \\
\text { POD; and } 2 \mathrm{mg} \text { twice on } 3 \text { POD } \\
\text { Placebo in similar manner, type } \\
\text { unclear } \\
\text { Intraop. and postop. }\end{array}$ & $\begin{array}{l}\text { Hospital: morphine } 0.1 \mathrm{mg} \\
\mathrm{kg}^{-1} \text { i.v. (max } 4 \text { doses) } \\
\text { Home: hydromorphone } 1 \\
\text { mg p.o. (max } 20 \mathrm{mg} \text { ), } 0-4 \\
\text { POD }\end{array}$ & 7 & $\begin{array}{l}\text { Dexamethasone reduced VASs on } 2 \text { POD ( } P=0.047 \text { ), other days NS } \\
\text { Hydromorphone consumption, NS }\end{array}$ \\
\hline $\begin{array}{l}\text { Vaiman and } \\
\text { colleagues } \\
\text { (2011) }\end{array}$ & $30 / 30$ & $\begin{array}{l}\text { Dexamethasone } 20 \mathrm{mg} \text { i.v. } \\
\text { Saline i.v. } \\
20 \text { h postop. }\end{array}$ & $\begin{array}{l}\text { Not given } 14-20 \mathrm{~h} \text { (type or } \\
\text { route unclear) }\end{array}$ & 1 & $\begin{array}{l}\text { Dexamethasone decreased VASs (postdrug us predrug), } P=0.022 \text {, } \\
\text { while placebo did not. Dexamethasone also decreased } \\
\text { postdrug-VASs us placebo }(P<0.05) \\
\text { Dexamethasone decreased muscle reactions and normalised } \\
\text { deglutition pattern (sEMG), while placebo did not }(P<0.05)\end{array}$ \\
\hline $\begin{array}{l}\text { Vaiman and } \\
\text { colleagues } \\
(2011)\end{array}$ & $30 / 30 / 30$ & $\begin{array}{l}\text { Dexamethasone } 20 \mathrm{mg} \text { i.v. } \\
\text { infusion } \\
\text { Oxycodone } 2 \mathrm{mg} \mathrm{h} \mathrm{h}^{-1} \text { i.v. } \\
\text { infusion ( } 14 \mathrm{mg} \text { ) } \\
\text { Saline i.v. infusion } \\
20 \mathrm{~h} \text { postop. } \\
\text { Oxycodone and placebo: } \\
\text { infusion } 16-23 \text { h postop. }\end{array}$ & $\begin{array}{l}\text { After the EMG test was } \\
\text { performed at } 24 \mathrm{~h} \text { (type } \\
\text { or route unclear) }\end{array}$ & 1 & $\begin{array}{l}\text { Oxycodone and dexamethasone decreased VASs (postdrug us } \\
\text { predrug) } P<0.05 \text {, while placebo did not. Oxycodone decreased } \\
\text { postdrug-VASs vs placebo }(P<0.05) \text {, dexamethasone did not. } \\
\text { Oxycodone decreased muscle reactions }(\text { sEMG), while } \\
\text { dexamethasone and placebo did not }(P<0.05) \text {. Oxycodone and } \\
\text { dexamethasone, in case of oedema, decreased dysphagia } \\
(P<0.05)\end{array}$ \\
\hline $\begin{array}{l}\text { Thimmasettaiah } \\
\text { and } \\
\text { Chandrappa }{ }^{42} \\
\text { (2012) }\end{array}$ & $25 / 25 / 25 / 25$ & $\begin{array}{l}\text { Dexamethasone } 0.5 \mathrm{mg} \mathrm{kg}^{-1} \text { i.v. } \\
\text { saline I.V. preop. (after inserting } \\
\text { i.v. cannula) us intraop. vs } \\
\text { postop. (PACU) } \\
\text { placebo: intraop. }\end{array}$ & $\begin{array}{l}\text { PACU: Tramadol } \mathrm{mg} \mathrm{kg}^{-1} \\
\text { i.v. if } \mathrm{VAS}>6\end{array}$ & 1 & $\begin{array}{l}\text { Dexamethasone decreased VAS at } 6 \mathrm{~h}(P<0.05) \text {, at } 12 \mathrm{~h}(P<0.001) \text {, } \\
\text { and at } 24 \mathrm{~h}(P<0.001) \text {. Preop. and intraop. groups NS. } \\
\text { Dexamethasone reduced } n \text { of tramadol doses } 0-24 \mathrm{~h}(P<0.05)\end{array}$ \\
\hline $\begin{array}{l}\text { Khafagy and } \\
\text { Osman } \\
\text { (2013) }\end{array}$ & $43 / 31$ & $\begin{array}{l}\text { Dexamethasone } 0.3 \mathrm{mg} \mathrm{kg}^{-1} \text { i.v. } \\
\text { (max } 8 \mathrm{mg} \text { ) } \\
\text { Saline i.v. } \\
\text { Intraop. }\end{array}$ & Unclear & 7 & $\begin{array}{l}\text { Dexamethasone reduced VAS on } 0 \text { POD and on } 4-7 \text { POD }(P<0.05) \text {. } \\
\text { Dexamethasone reduced overall VAS during 0-7 POD } \\
(P=0.002) \text {. 1st liquid intake NS. 1st solid food intake: } \\
\text { dexamethasone earlier us control group }(P=0.05)\end{array}$ \\
\hline $\begin{array}{l}\text { Mikkelsen and } \\
\text { colleagues }^{44} \\
(2006)\end{array}$ & $22 / 27$ & $\begin{array}{l}\text { Gabapentin } 1200 \mathrm{mg} \text { p.o. } 1 \mathrm{~h} \\
\text { preop., } 600 \mathrm{mg} \text { p.o. twice on } \\
\text { 0 POD, } 600 \mathrm{mg} \text { p.o. three times } \\
\text { on } 1-5 \text { POD } \\
\text { Placebo in similar manner, type } \\
\text { unclear } \\
\text { Preop. and postop. }\end{array}$ & $\begin{array}{l}\text { Morphine } 2.5 \text { mg i.v., } 0-4 \mathrm{~h} \\
\text { Home: ketobemidone } 2.5 \\
\text { mg p.o., 0-5 POD }\end{array}$ & 5 & $\begin{array}{l}\text { VRSr and VASs } 2-4 \mathrm{~h} \text { and } 1-5 \text { POD, NS } \\
\text { Total dose of morphine } 0-4 \mathrm{~h} \text { NS } \\
\text { Gabapentin reduced total dose of ketobemidone } 0-24 \mathrm{~h} \text {, on } \\
\text { following days NS }\end{array}$ \\
\hline $\begin{array}{l}\text { Jeon and } \\
\text { colleagues }{ }^{45} \\
(2009)\end{array}$ & $32 / 26$ & $\begin{array}{l}\text { Gabapentin } 600 \mathrm{mg} \text { p.o. on } \\
\text { previous evening and } 600 \mathrm{mg} \\
\text { p.o. } 1 \text { h preop. } \\
\text { Similar capsule p.o. } \\
\text { Preop. }\end{array}$ & $\begin{array}{l}\text { PCA fentanyl } 20 \mu \mathrm{g} \text { bolus, } \\
\text { diclofenac } 75 \mathrm{mg} \text { i.m., } 0 \\
-2 \text { POD }\end{array}$ & 9 & $\begin{array}{l}\text { Gabapentin reduced VASs at } 2 \text { and } 4 \mathrm{~h}(P=0.04, P=0.04) \text {, on } \\
\text { following POD NS. VASr } 0-7 \text { POD NS } \\
\text { Gabapentin reduced total dose of PCA fentanyl }(P=0.002) \text {, and } \\
\text { total dose of diclofenac on } 0-2 \text { POD at hospital }(P=0.001) \text {. } \\
\text { Patient satisfaction NS }\end{array}$ \\
\hline $\begin{array}{l}\text { Abdelmageed } \\
\text { and } \\
\text { colleagues }{ }^{46} \\
(2010)\end{array}$ & $30 / 30$ & $\begin{array}{l}\text { Gabapentin } 1200 \text { mg p.o. } \\
\text { Placebo tablets, type unclear } \\
2 \text { h preop. }\end{array}$ & $\begin{array}{l}\text { Pethidine } 1 \mathrm{mg} \mathrm{kg}^{-1} \text { i.m. } \\
\text { every } 6 \mathrm{~h} \text { if } \mathrm{VAS} \geq 3 \text { or if } \\
\text { needed }\end{array}$ & 1 & $\begin{array}{l}\text { Gabapentin reduced VAS at all time points: } 1,3,6,12,18,24 \mathrm{~h} \\
(P<0.001) \text {. Gabapentin decreased worst VAS }(P<0.001) \\
\text { Gabapentin reduced total dose of pethidine } 0-24 \mathrm{~h}(P<0.001) \\
\text { and increased time to } 1 \text { st dose of pethidine }(P<0.001)\end{array}$ \\
\hline
\end{tabular}




\begin{tabular}{|c|c|c|c|c|c|}
\hline $\begin{array}{l}\text { Reference (and } \\
\text { year) }\end{array}$ & $\begin{array}{l}\text { No. of patients, } \\
\text { active/placebo }\end{array}$ & $\begin{array}{l}\text { Study analgesics } \\
\text { Study arm } \\
\text { Placebo } \\
\text { Time of administration }\end{array}$ & $\begin{array}{l}\text { Rescue analgesic, time of } \\
\text { administration }\end{array}$ & $\begin{array}{l}\text { Duration of } \\
\text { follow-up } \\
\text { (days) }\end{array}$ & Analgesic outcome results (study drug vs placebo) \\
\hline $\begin{array}{l}\text { Mathiesen and } \\
\text { colleagues } \\
\text { (2011) }\end{array}$ & $43 / 45$ & $\begin{array}{l}\text { Pregabalin } 300 \text { mg p.o. } \\
\text { Placebo, type unclear } \\
1 \text { h preop. }\end{array}$ & $\begin{array}{l}\text { Morphine } 2.5 \mathrm{mg} \text { i.v., } 0-1 \mathrm{~h} \\
\text { ketobemidone } 2.5 \mathrm{mg} \\
\text { p.o., } 0-24 \mathrm{~h}\end{array}$ & 1 & $\begin{array}{l}\text { Pregabalin reduced VASs at } 2 \text { and } 4 \mathrm{~h}(P=0.009, P<0.003) \\
\text { Pregabalin also reduced VASs mean } 2-24 \mathrm{~h}(P=0.009) \text { and VASr } \\
\text { at } 4 \mathrm{~h}(P=0.03) \text {. Pregabalin reduced total dose of ketobemidone } \\
1-4 \mathrm{~h}(P=0.003), 1-24 \mathrm{~h} \text { NS. Total dose of morphine } 0-1 \mathrm{~h} \text { NS }\end{array}$ \\
\hline $\begin{array}{l}\text { Kawamata and } \\
\text { colleagues }{ }^{48} \\
(1998)\end{array}$ & $12 / 12 / 12$ & $\begin{array}{l}\text { Dextromethorphan } 45 \mathrm{mg} \text { p.o. } \\
\text { Dextromethorphan } 30 \mathrm{mg} \text { p.o. } \\
\text { Starch tablet p.o. } \\
1 \mathrm{~h} \text { preop. }\end{array}$ & $\begin{array}{l}\text { Diclofenac } 50 \text { mg p.r., 0-6 } \\
\text { POD }\end{array}$ & 7 & $\begin{array}{l}\text { Dextromethorphan } 45 \mathrm{mg} \text { decreased VASs and VASr on all } 0-6 \\
\text { POD us placebo }(P<0.05) \text {. Dextromethorphan } 30 \mathrm{mg} \text { decreased } \\
\text { VASs on } 0 \text { POD and VASr on } 0,1 \text {, and } 6 \text { POD }(P<0.05) \text {. } \\
\text { Dextromethorphan } 45 \mathrm{mg} \text { and } 30 \mathrm{mg} \text { reduced total dose of } \\
\text { diclofenac/patient }(P<0.05, P<0.05) \text {. Dextromethorphan } \\
\text { increased time to } 1 \text { st dose of diclofenac }(P<0.05)\end{array}$ \\
\hline $\begin{array}{l}\text { Rafiei and } \\
\text { colleagues }{ }^{49} \\
\text { (2012) }\end{array}$ & $20 / 20$ & $\begin{array}{l}\text { Dextromethorphan } 45 \mathrm{mg} \text { p.o. } \\
\text { Placebo tablet p.o. } \\
1 \text { h preop. }\end{array}$ & $\begin{array}{l}\text { Pethidine } 0.5 \mathrm{mg} \mathrm{kg}^{-1} \text { i.v. if } \\
\text { VAS } \geq 3\end{array}$ & 1 & $\begin{array}{l}\text { Dextromethorphan increased pain-free time period }(P=0.002) \text {, } \\
\text { and decreased VASs } 0-24 \mathrm{~h}(P=0.047) \text {, VASr NS. } \\
\text { Dextromethorphan increased time to } 1 \text { st dose of pethidine } \\
(P=0.005) \text { and reduced total dose of pethidine/patient }(P=0.005)\end{array}$ \\
\hline $\begin{array}{l}\text { Van Elstraete } \\
\text { and } \\
\text { colleagues } \\
\text { (2004) }\end{array}$ & $20 / 20$ & $\begin{array}{l}\text { Ketamine } 0.5 \text { mg kg-1 i.v. bolus } \\
\text { with ketamine } 2 \mu \mathrm{kg}^{-1} \mathrm{~min}^{-1} \\
\text { infusion saline i.v. bolus with } \\
\text { i.v. infusion } \\
\text { Bolus at induction with infusion } \\
\text { until end of procedure }\end{array}$ & $\begin{array}{l}\text { PACU: Morphine } 3 \mathrm{mg} \text { i.v. } \\
\text { every } 5 \text { min until VAS }<30 \\
\text { Ward: morphine } 10 \mathrm{mg} \\
\text { p.o. every } 4 \text { h until } \\
\text { VASs }<30 \\
\text { Ketoprofen } 100 \mathrm{mg} \text { p.o. } \\
\text { and paracetamol } 500 \mathrm{mg} \\
\text { p.o., } 0-24 \mathrm{~h}\end{array}$ & 1 & $\begin{array}{l}\text { Total dose of morphine/patient NS. } \\
\text { Time to 1st morphine dose at the ward NS. } \\
\text { VASr, VASs NS }\end{array}$ \\
\hline $\begin{array}{l}\text { Vaiman and } \\
\text { Krakovski } \\
\text { (2012) }\end{array}$ & $30 / 30$ & $\begin{array}{l}\text { Oxycodone } 2 \mathrm{mg} \mathrm{h}^{-1}(14 \mathrm{mg}) \text { i.v. } \\
\text { infusion } \\
\text { Saline i.v. infusion } \\
16-23 \text { h postop. }\end{array}$ & Unclear & 1 & $\begin{array}{l}\text { Oxycodone decreased VASs (postdrug us predrug), } P=0.03 \text {, while } \\
\text { placebo did not. Oxycodone decreased postdrug VASs } \\
\text { (oxycodone us placebo, } P<0.05 \text { ) and muscle reactions (sEMG), } \\
P=0.03\end{array}$ \\
\hline
\end{tabular}


Table 2 Reported pain intensity values during follow-up time. ${ }^{*}$ pain intensity values not available, only need of rescue analgesics reported; $\boldsymbol{\Delta}=$ pain relief; $\boldsymbol{\square}=\mathrm{VAS} / \mathrm{VRS} / \mathrm{NRS}$, $\mathrm{O}=\mathrm{VASs} /$ VRSs/NRSs; - VASr/VRSr/NRSr and VASs/VRSs/NRSs. NMDA, N-methyl-D-aspartate; NRS,; NRSr,; NRSs,; pod, postoperative day; VAS, visual analogue scale; VASr, VAS at rest; VASs, VAS on swallowing; VRS, verbal rating scale; VRSr, VRS at rest; VRSs, VRS on swallowing; NRS, numeric rating scale; NRSr, NRS at rest; NRSs, NRS at swallowing

\begin{tabular}{|c|c|c|c|c|c|c|c|c|c|c|c|c|c|c|}
\hline Study (and year) & $1 \mathrm{~h}$ & $2 \mathrm{~h}$ & $4 \mathrm{~h}$ & $12 \mathrm{~h}$ & $24 \mathrm{~h}$ & 2 pod & 3 pod & 4 pod & 5 pod & 6 pod & 7 pod & 8 pod & 9 pod & 10 pod \\
\hline \multicolumn{15}{|l|}{ Paracetamol } \\
\hline Atef and Fawaz ${ }^{23}(2008)$ & - & - & - & & - & & & & & & & & & \\
\hline Salonen and colleagues ${ }^{24}(2009)$ & $\bullet$ & $\bullet$ & • & & & & & & & & & & & \\
\hline \multicolumn{15}{|l|}{ NSAIDs } \\
\hline Parker and colleagues ${ }^{25}$ (1986), ibuprofen, acetylsalicylic acid & $\Delta$ & $\Delta$ & $\Delta$ & & $\boldsymbol{\Delta}$ & $\Delta$ & $\Delta$ & $\Delta$ & $\Delta$ & & & & & \\
\hline Rorarius and colleagues ${ }^{26}$ (1993), indomethacin, diclofenac & * & $*$ & & & & & & & & & & & & \\
\hline Tarkkila and Saarnivaara ${ }^{27}(1999)$, ketoprofen & & - & & & - & & & & & & & & & \\
\hline Salonen and colleagues ${ }^{28}$ (2001), ketoprofen & - & - & - & & - & & & & & & & & & \\
\hline Naesh and colleagues ${ }^{29}$ (2005), rofecoxib & & & घ & a & $\mathbf{a}$ & & & & & & & & & \\
\hline Ismail and Mowafi ${ }^{30}$ (2010), lornoxicam & - & - & $\bar{\bullet}$ & $\bar{\bullet}$ & 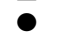 & & & & & & & & & \\
\hline Mowafi and colleagues $^{31}$ (2011), lornoxicam & - & - & - & - & - & & & & & & & & & \\
\hline Xie and colleagues ${ }^{32}$ (2012), parecoxib & - & - & - & - & - & & & & & & & & & \\
\hline \multirow{2}{*}{\multicolumn{15}{|c|}{ Dexamethasone }} \\
\hline & & & & & & & & & & & & & & \\
\hline Fields and colleagues ${ }^{34}$ (1994) & & & 口 & a & 口 & $\mathbf{a}$ & $\mathbf{\square}$ & $\mathbf{\square}$ & $\mathbf{\square}$ & $\mathbf{\square}$ & घ & & & \\
\hline Carr and colleagues ${ }^{35}$ (1999) & & & & & $\overline{0}$ & 0 & 0 & 0 & 0 & 0 & 0 & 0 & 0 & 0 \\
\hline Stewart and colleagues ${ }^{36}(2002)$ & & & & 0 & 0 & 0 & 0 & 0 & 0 & 0 & 0 & 0 & 0 & \\
\hline Al-Shehri ${ }^{37}(2004)$ & & & & & $\mathbf{\square}$ & $\mathbf{\square}$ & $\mathbf{\square}$ & $\mathbf{\square}$ & $\mathbf{a}$ & घ & घ & $\mathbf{\square}$ & 口 & \\
\hline Rujirojindakul and colleagues ${ }^{38}$ (2008) & a & & $\mathbf{a}$ & & - & $\mathbf{\square}$ & & & & & & & & \\
\hline Lachance and colleagues $^{39}(2008)$ & & & & 0 & $\overline{0}$ & $\overline{0}$ & 0 & 0 & 0 & 0 & & & & \\
\hline Vaiman and colleagues ${ }^{40}(2011)$ & & & & & 0 & & & & & & & & & \\
\hline Vaiman and colleagues ${ }^{41}(2011)$ & & & & & 0 & & & & & & & & & \\
\hline Thimmasettaiah and Chandrappa $^{42}$ (2012) & & & & a & a & & & & & & & & & \\
\hline Khafagy and Osman ${ }^{43}(2013)$ & & & & & $\overline{\mathbf{u}}$ & & 口 & 口 & [ & [ & [ & & & \\
\hline \multicolumn{15}{|l|}{ Gabapentinoids } \\
\hline Mikkelsen and colleagues ${ }^{44}$ (2006), gabapentin & & & - & & $\bullet$ & $\bullet$ & - & - & - & & & & & \\
\hline Jeon and colleagues ${ }^{45}(2009)$, gabapentin & $\bullet$ & $\bullet$ & $\bullet$ & $\bullet$ & - & - & - & - & - & - & - & - & - & \\
\hline Abdelmageed and colleagues ${ }^{46}(2010)$, gabapentin & [ & & & $\boldsymbol{\square}$ & $\mathbf{a}$ & & & & & & & & & \\
\hline Mathiesen and colleagues ${ }^{47}$ (2011), pregabalin & & - & - & & - & & & & & & & & & \\
\hline NMDA antagonists & & & & & & & & & & & & & & \\
\hline Kawamata and colleagues ${ }^{48}$ (1998), dextromethorphan & - & & & & • & $\bullet$ & $\bullet$ & $\bullet$ & & $\bullet$ & & & & \\
\hline Rafiei and colleagues ${ }^{49}$ (2012), dextromethorphan & & & & & 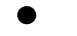 & & & & & & & & & \\
\hline Van Elstraete and colleagues ${ }^{50}$ (2004), ketamine & $\bullet$ & $\bullet$ & $\bullet$ & $\bullet$ & $\bullet$ & & & & & & & & & \\
\hline Vaiman and Krakovski ${ }^{51}$ (2012), oxycodone & & & & & 0 & & & & & & & & & \\
\hline
\end{tabular}




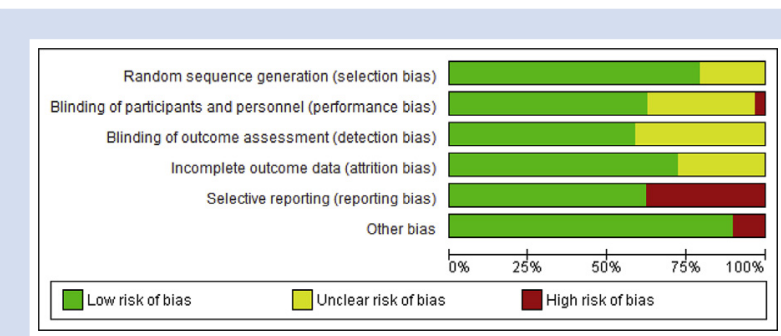

Fig 2. Risk of bias graph. Review authors' judgments about each risk of bias item presented as percentages across all included studies.

In other NSAID studies, the reporting of pain intensities was unsuitable for meta-analysis (pain relief, ${ }^{25}$ median values instead of mean ${ }^{29-33}$ ) and the results are presented descriptively. In a study ${ }^{25}$ with oral ibuprofen $600 \mathrm{mg}$, acetylsalicylic acid $600 \mathrm{mg}$, or placebo 0-4 times daily for 6 postoperative days in patients with moderate to severe post-tonsillectomy pain, pain relief within $24 \mathrm{~h}$ after initial treatment was faster with ibuprofen compared with placebo. Acetylsalicylic acid did not have any effect on pain outcomes. In a study ${ }^{26}$ of diclofenac $75 \mathrm{mg}$ i.v. and indomethacin $50 \mathrm{mg}$ i.v. intraoperatively, indomethacin reduced the need for opioids at the PACU, while diclofenac had no effect.

Cyclooxygenase-2 selective NSAIDs, lornoxicam $16 \mathrm{mg}$ i.v. before operation in two studies ${ }^{30,31}$ and parecoxib $40 \mathrm{mg}$ i.v. intraoperatively and after operation ${ }^{32}$ reduced pain and the

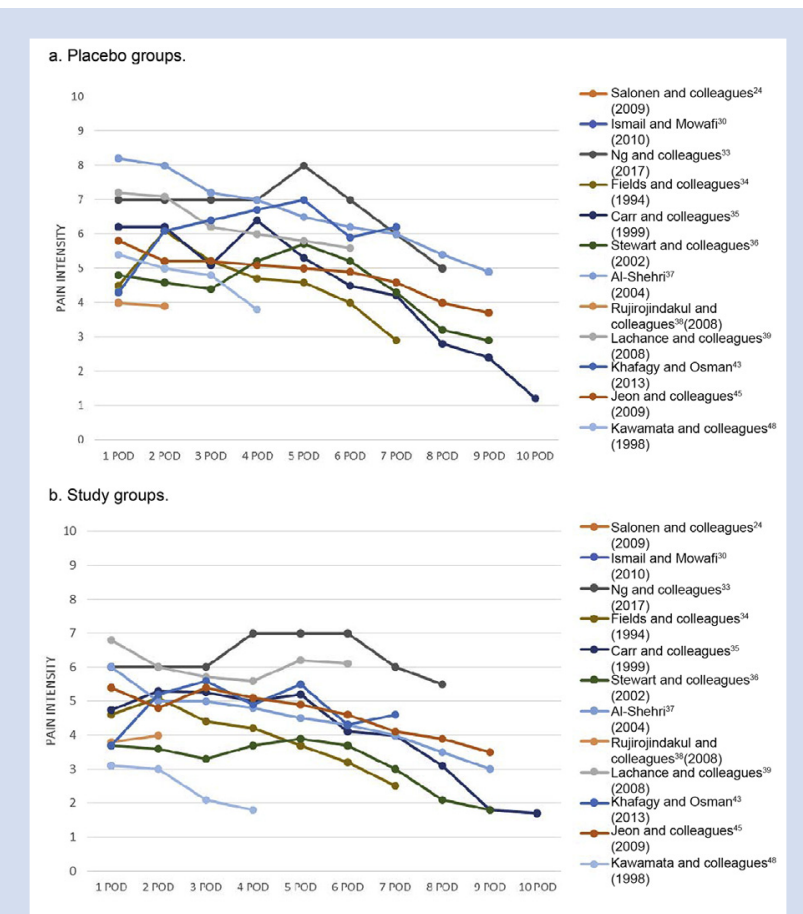

Fig 3. Pain intensities during 1-9 postoperative days (POD). (a) Placebo groups; (b) study groups. need for rescue analgesia within $24 \mathrm{~h}$. Oral rofecoxib $50 \mathrm{mg}$ before operation decreased the incidence of pain (VAS $>5$ ) and the need for opioids within the first 8 h. ${ }^{29}$ Celecoxib $200 \mathrm{mg}$ twice daily for 1-10 postoperative days with additional paracetamol $1 \mathrm{~g}$ four times daily did not reduce pain or need for opioids. $^{33}$

The majority of patients given NSAIDs $(74 \%)$ or placebo (85\%) needed rescue analgesia within $24 \mathrm{~h}$ after the operation (relative risk [RR] 0.91 [95\% CI 0.79-1.05], $P=0.21$ )..$^{27,28,30-32} \mathrm{Six}$ studies $^{27,28,30-33}$ reported incidence of PTH; none found an increased risk.

Randomised studies comparing dexamethasone with placebo

Ten studies ${ }^{34-43}$ including 590 patients are summarised in Table 1 (Supplementary Appendix A1). Pooled estimates demonstrated that single-dose dexamethasone of $8 \mathrm{mg}$ to $0.5 \mathrm{mg} \mathrm{kg}^{-1}$ decreased pain intensity equivalent to $23 \%$ at 4 h $(-1.40$ [95\% CI -1.64 to -1.16$\left.], P<0.001, I^{2}=0 \%\right)$ but no longer at $24 \mathrm{~h}(\mathrm{P}=0.05)$ (Fig. 4, Supplementary Appendix S4). When the only multiple-dose study ${ }^{37}$ suitable for metaanalysis was included, a significant $17 \%$ reduction at $24 \mathrm{~h}$ was observed, although with a high heterogeneity $\left(\mathrm{I}^{2}=93 \%\right)$. In two studies with high single doses of dexamethasone (20 $\mathrm{mg}^{35}$ and $0.5 \mathrm{mg} \mathrm{kg}^{-142}$ ), the need for rescue opioids decreased in the PACU. Reduction of pain intensity lasted beyond the first postoperative day in all multiple-dose studies and in one single-dose study. ${ }^{36,37,39,43}$ The total dose of rescue analgesic (combination of paracetamol 500 $\mathrm{mg}$ and codeine $16 \mathrm{mg}$ ) was reduced over $2-7$ postoperative days in a study on dexamethasone for 8 postoperative days. $^{36}$

Pooled estimates demonstrated that dexamethasone was associated with less PONV during 24-48 h after operation (RR 0.41 [95\% CI 0.29-0.59], $\left.P<0.001, I^{2}=0 \%\right)^{35,36,38,42}$ without increase in risk for PTH (RR 0.76 [95\% CI 0.37-1.56], $P=0.46$, $\left.\mathrm{I}^{2}=0 \%\right)^{35,36,38,42}$ (four studies reported risk for PTH).

Randomised studies comparing gabapentinoids with placebo

Four studies including 255 patients (three gabapentin ${ }^{44-46}$ and one pregabalin ${ }^{47}$ ) are summarised in Table 1 (and Supplementary Appendix S1). Pooled estimates demonstrated that gabapentinoids decreased pain intensity equivalent to $30 \%$ at $4 \mathrm{~h}(-1.58$ [95\% CI -2.28 to -0.88$\left.], \mathrm{P}<0.001, \mathrm{I}^{2}=0 \%\right)$ and equivalent to $13 \%$ at $24 \mathrm{~h}(-1.03$ [95\% CI -1.30 to -0.77 ], $\mathrm{P}<0.001, \mathrm{I}^{2}=0 \%$ ) (Fig. 4, Supplementary Appendix S5). Gabapentin $300-2400 \mathrm{mg}$ on the day of operation reduced the need for rescue analgesics (opioids or diclofenac) within $24 \mathrm{~h} .{ }^{44-46}$ Pregabalin $300 \mathrm{mg}$ reduced the need for opioids within $4 \mathrm{~h}^{47}$ In one study ${ }^{44}$ with high and multiple doses of gabapentin, adverse events were common and outweighed beneficial effects. Gabapentin decreased postoperative vomiting in pooled analysis estimation, most likely because of the decreased need for rescue opioids (RR 0.55 [95\% CI 0.32-0.95], $P=0.03, \mathrm{I}^{2}=2 \%$ ).

\section{Randomised studies comparing NMDA receptor antagonists with placebo}

Three studies (116 patients) on NMDA receptor antagonists (two dextrometorphan ${ }^{48,49}$ and one ketamine ${ }^{50}$ ) are summarised in Table 1 (Supplementary Appendix S1). Ketamine ${ }^{50}$ and 


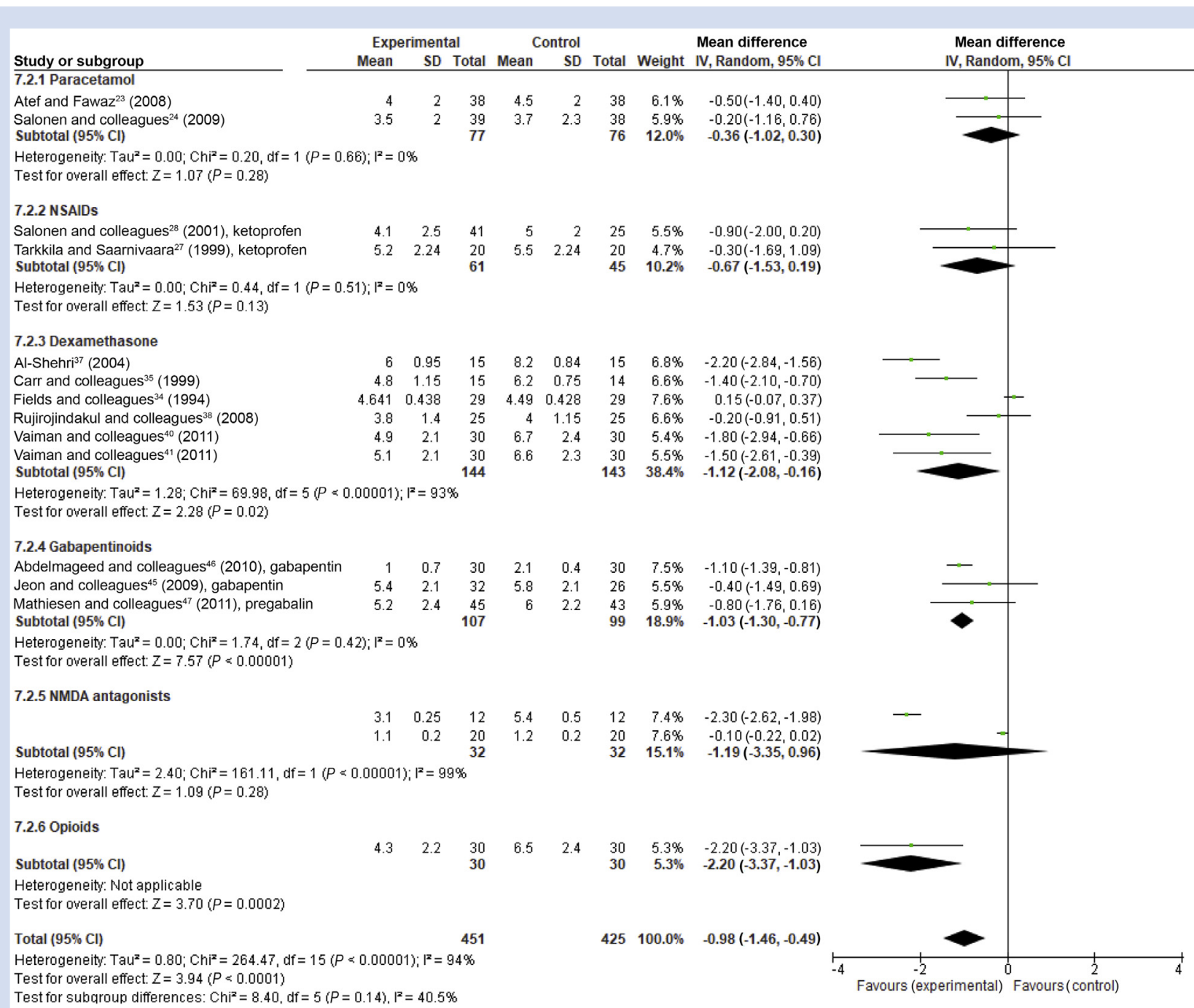

Fig 4. Forest plot showing the effect of perioperatively administered analgesics and dexamethasone on pain intensity at $24 \mathrm{~h}$. Pain intensity values (scale $0-10$ ) with confidence intervals (CI). Data evaluated using a random effects model.

dextromethorphan studies ${ }^{48,49}$ were too heterogeneous to be combined in pooled analysis. Dextromethorphan $45 \mathrm{mg}$ reduced VASs and the total dose of rescue analgesics within 24 $\mathrm{h}$, and prolonged time to first request of rescue analgesics in both studies. ${ }^{48,49}$ In the study of Kawamata and colleagues, ${ }^{48}$ dextromethorphan reduced pain intensity over the follow-up period of 6 postoperative days.

Ketamine as an intraoperative bolus and infusion failed to reduce pain intensity and total dose of opioids, and did not prolong the time to first analgesic requests over a follow-up period of $24 \mathrm{~h} .{ }^{50}$ Ketamine did not increase the incidence of adverse events; nausea and sedation scores were similar between the study groups and none experienced hallucinations or nightmares.

Randomised studies comparing opioids with placebo One study on oxycodone ${ }^{51}$ including 60 patients is summarised in Table 1 (and Supplementary Appendix S1). I.V. oxycodone $2 \mathrm{mg} \mathrm{h}^{-1}$ decreased pain intensity at $24 \mathrm{~h}$ after operation. Data on rescue analgesics or adverse events were not reported.

\section{Discussion}

\section{Summary of evidence}

The main finding of this review was the scarcity of data and short duration of follow-up in studies that investigated analgesics for post-tonsillectomy pain. This finding is clinically relevant considering the number of tonsillectomies performed yearly and the intensity of pain after the procedure. I.V. paracetamol reduced pain intensity at $3-4 \mathrm{~h}$ and the need for opioids $\leq 24 \mathrm{~h}$ after surgery. Ketoprofen at varying doses (100 $\mathrm{mg}$ or $0.5 \mathrm{mg} \mathrm{kg}^{-1}$ i.v.) failed to decrease pain intensity within $24 \mathrm{~h}$. However, both studies reported a reduced need for opioids within $24 \mathrm{~h}$. In other NSAID studies, pooled analysis was not possible because of the large heterogeneity. In individual studies, NSAIDs administered on the day of operation reduced 
pain intensity, the need for rescue analgesics, or both within $24 \mathrm{~h}$. Celecoxib on postoperative days 1-10 had no effect on pain intensity or the need for opioids. In all NSAID studies, the majority of patients needed rescue analgesics, thus indicating that NSAIDs alone do not provide adequate analgesia for tonsillectomy patients.

Dexamethasone as a single intraoperative dose reduced pain within $4 \mathrm{~h}$. When administered in multiple doses, the effect lasted beyond the first postoperative day. Gabapentinoids and dextromethorphan decreased pain intensity and need of rescue analgesia within 24 hours. Ketamine failed to show any effect at the studied dose.

Because of the high post-tonsillectomy pain intensity, the analgesic effect did not reach clinical meaningfulness when any of the analgesics or dexamethasone was used alone. PONV was reduced by dexamethasone and gabapentinoids over 24-48 $\mathrm{h}$ after operation because of antiemetic and opioidsparing effects. The incidence of PTH was reported in paracetamol, NSAID and dexamethasone studies; no increase in the risk for PTH was found. Serious adverse events were not reported.

\section{Limitations}

A systematic review and meta-analysis combine data to estimate treatment effects more precisely than is possible in single studies. However, the accuracy of results of systematic reviews depends on the availability and quality of the data available. A thorough search was performed, and all relevant studies were likely included in the study. Only studies in English were included, which could limit the accuracy of results; however, no evidence of a systematic bias from the use of English language restriction in systematic review-based metaanalyses in conventional medicine has been found. ${ }^{20}$ Funnel plots of the results were drawn and examined in the review group and did not show any apparent publication bias among studies.

Studies were clinically heterogeneous with varying timing of study medications, use of rescue and additional analgesics, and duration of follow-up. Therefore, combining results in meta-analysis was possible only in selected studies and only for pain intensity. Although there were sub-optimally only two studies in some analgesic groups, we conducted a metaanalysis also for them to allow rough comparison between groups.

In the present study, 12 out of 29 included trials failed to report the primary outcome thus presenting a risk for bias.

The main limitation was the short study and follow-up time, especially in the NSAID studies. From the patient point of view, adequate pain management at home is important. At hospital, pain can be easily treated with opioids, but problems arise after discharge when strong opioids are not available. There are minimal data on optimal analgesics for posttonsillectomy pain at home.

All studies did not report pain intensities at rest and on swallowing, thus decreasing the accuracy of the results. Pain on movement (swallowing) should be reported, as it is a major determinant of how well patients can drink and eat at home during recovery.

\section{Comparison with results of previous studies}

In a Cochrane review (that included the two studies also included in this review), a single dose of either paracetamol or propacetamol i.v. was found to provide approximately $4 \mathrm{~h}$ of effective analgesia for about $36 \%$ of patients with acute postoperative pain. ${ }^{8,23,24}$ This is consistent with our findings that paracetamol 1-2 g i.v. intraoperatively decreased pain intensity at 3-4 h. A reduced need for opioids within $24 \mathrm{~h}$ was reported in both studies with a cumulative dose of paracetamol 4 g i.v. ${ }^{23}$ and a single dose of paracetamol 2 g i.v. ${ }^{24}$

NSAIDs are widely used for postoperative pain and are effective for various types of surgical pain. ${ }^{52,53}$ We found no previous reviews of NSAIDs for post-tonsillectomy pain in adults. Consistent with previous reports, NSAIDs reduced pain intensity or need for rescue analgesics in most studies.

A review on perioperative single-dose dexamethasone for postoperative pain ${ }^{14}$ (that included one study ${ }^{35}$ also included here) reported reduced pain intensity at 4 and $24 \mathrm{~h}$ and opioid consumption at a high dose of dexamethasone (more than 0.1 $\mathrm{mg} \mathrm{kg}^{-1}$ ). A review on dexamethasone and post-tonsillectomy morbidity ${ }^{15}$ (that included five studies ${ }^{34-37,39}$ also included here) reported reduced pain intensity on the first postoperative day with high-dose $(>10 \mathrm{mg})$ steroids $(P<0.001)$. Our results in the present review are comparable. We did not observe an increased risk for PTH associated with the use of dexamethasone. Two recent studies (a register study and a systematic review) indicate an increased risk for reoperation as a result of PTH in children, but not in adults. ${ }^{54,55}$

Previous reviews on perioperative gabapentinoids have found a reduction of postoperative pain, opioid consumption, and opioid-related adverse effects. ${ }^{5,18}$ A recent systematic review that analysed the benefit of pregabalin for acute pain reported a minimal opioid-sparing effect in studies with low overall risk of bias; this review included two tonsillectomy studies, of which one was also included in our analysis. ${ }^{47}$ We found similar analgesic effects in our study; gabapentinoids reduced pain at 4 and $24 \mathrm{~h}$ after operation in pooled analysis. However, regarding observed pain reduction at $24 \mathrm{~h}$, one study ${ }^{46}$ with an unclear overall risk for bias was responsible for the majority of the effect ( $60 \%$ weight). When an analysis that excluded this study was performed, there was no significant reduction in pain intensity. Gabapentin 300-1200 mg before operation reduced the need for opioids within 4 and 24 $\mathrm{h}^{44-46}$ and pregabalin $300 \mathrm{mg}$ before operation reduced this need within 4 h. ${ }^{47}$ Previous results on adverse effects associated with gabapentinoids compared with placebo are conflicting. ${ }^{5,18}$ In the present study, the risk of adverse effects was not increased except in one high-dose study, where dizziness, vomiting, and gait disturbance were more frequent in the study group. ${ }^{44}$

Dextromethorphan is an NMDA receptor antagonist and is a widely used antitussive medication. Dextromethorphan has been shown to potentiate the antinociceptive effects of opiates and prevent pain sensitivity and opioid tolerance without significant side-effects. ${ }^{56,57}$ A review of 28 studies on perioperative dextromethorphan (including one study ${ }^{48}$ also included here) revealed a reduced need for opioids and considered perioperative dextromethorphan as a safe adjuvant agent to opioid analgesia. ${ }^{57}$ In our study, dextromethorphan $45 \mathrm{mg}$ before operation decreased pain intensity over 24 $\mathrm{h}$ and total dose and time to first rescue analgesic in both studies. ${ }^{48,49}$ Ketamine is a well-known NMDA receptor antagonist and when used in subanaesthetic doses can reduce opioid requirements, pain sensitivity, or both in acute postoperative pain with mild or absent adverse effects (Cochrane review 2005). ${ }^{58}$ In a systematic review (2014) in paediatric tonsillectomies, i.v. ketamine before operation showed an 
opioid-sparing effect and a reduction of pain intensity and time to resumption of liquid diet. ${ }^{59}$ Adverse effects such as nausea and vomiting, sedation, bad dreams, sleep pattern change, or hallucinations were absent. In the present review, the only ketamine study included failed to be effective in studied doses.

We did not find reviews on opioids for post-tonsillectomy pain. We included only one opioid study in which postoperative oxycodone infusion decreased pain intensity as expected.

\section{Conclusions and clinical implications}

This study confirmed the beneficial analgesic effects of paracetamol, NSAIDs, dexamethasone, gabapentinoids, and dextromethorphan for post-tonsillectomy pain on the day of operation. Dexamethasone in multiple doses had an analgesic effect that exceeded $24 \mathrm{~h}$. However, the use of steroids with high total doses or continued after the day of operation must be done with caution considering both the risks and benefits. Based on our results, gabapentin, pregabalin, and possibly dextromethorphan could be useful at moderate doses as adjuvants to other analgesics. Ketamine in subanaesthetic doses is effective in various types of surgeries in decreasing the need for opioids and pain sensitivity. Although ketamine was not effective in the one included study in our analysis, it may have efficacy for post-tonsillectomy pain; this should be studied in further trials.

The short follow-up times and clinical heterogeneity of studies limit the usefulness of the results, which should be interpreted with caution.

Single analgesics or dexamethasone alone do not provide a clinically meaningful analgesic effect for the treatment of post-tonsillectomy pain. Multimodal analgesia is thus required. Further studies are needed to identify the best possible combinations. Pain on swallowing should be used as a primary analgesic outcome and a follow-up time of 1-2 postoperative weeks is recommended.

\section{Authors' contributions}

Study design/planning: all authors

Extraction of data: HKT, AT, KH

Data analysis: HKT, VKK

Writing paper: HKT

Revising paper: all authors

\section{Declarations of interest}

The authors declare that they have no conflicts of interest.

\section{Appendix A. Supplementary data}

Supplementary data to this article can be found online at https://doi.org/10.1016/j.bja.2019.04.063.

\section{References}

1. Crowson MG, Ryan MA, Rocke DJ, Raynor EM, Puscas L. Variation in tonsillectomy rates by health care system type. Int J Pediatr Otorhinolaryngol 2017; 94: 40-4

2. Ovesen T, Kamarauskas G, Dahl M, Mainz J. Pain and bleeding are the main determinants of unscheduled contacts after outpatient tonsillectomy. Dan Med J 2012; 59: A4382

3. Lavy JA. Post-tonsillectomy pain: the difference between younger and older patients. Int J Pediatr Otorhinolaryngol 1997; 42: 11-5

4. Sarny S, Habermann W, Ossimitz G, Stammberger H. Significant post-tonsillectomy pain is associated with increased risk of hemorrhage. Ann Otol Rhinol Laryngol 2012; 121: 776-81

5. Toma AG, Blanshard J, Eynon-Lewis N, Bridger MW. Posttonsillectomy pain: the first ten days. J Laryngol Otol 1995; 109: 963-4

6. Patel HH, Straight CE, Lehman EB, Tanner M, Carr MM. Indications for tonsillectomy: a 10 year retrospective review. Int J Pediatr Otorhinolaryngol 2014; 78: 2151-5

7. Hamunen K, Kontinen V. Systematic review on analgesics given for pain following tonsillectomy in children. Pain 2005; 117: 40-50

8. McNicol ED, Ferguson MC, Haroutounian S, Carr DB, Schumann R. Single dose intravenous paracetamol or intravenous propacetamol for postoperative pain. Cochrane Database Syst Rev 2016; 23: CD007126

9. Ong CKS, Seymour RA, Lirk P, Merry AF. Combining paracetamol (acetaminophen) with nonsteroidal antiinflammatory drugs: a qualitative systematic review of analgesic efficacy for acute postoperative pain. Anesth Analg 2010; 110: 1170-9

10. Derry CJ, Derry S, Moore RA. Single dose oral ibuprofen plus paracetamol (acetaminophen) for acute postoperative pain. Cochrane Database Syst Rev 2013; 24: CD010210

11. Møiniche S, Rømsing J, Dahl JB, Tramèr MR. Nonsteroidal antiinflammatory drugs and the risk of operative site bleeding after tonsillectomy: a quantitative systematic review. Anesth Analg 2003; 96: 68-77

12. Riggin L, Ramakrishna J, Sommer DD, Koren GA. 2013 Updated systematic review \& meta-analysis of 36 randomized controlled trials; no apparent effects of non steroidal anti-inflammatory agents on the risk of bleeding after tonsillectomy. Clin Otolaryngol 2013; 38: 115-29

13. Lewis SR, Nicholson A, Cardwell ME, Siviter G, Smith AF. Nonsteroidal anti-inflammatory drugs and perioperative bleeding in paediatric tonsillectomy. Cochrane Database Syst Rev 2013; 18: CD003591

14. De Oliveira GS, Almeida MD, Benzon HT, McCarthy RJ. Perioperative single dose systemic dexamethasone for postoperative pain: a meta-analysis of randomized controlled trials. Anesthesiology 2011; 115: 575-88

15. Diakos EA, Gallos ID, El-Shunnar S, Clarke M, Kazi R, Mehanna H. Dexamethasone reduces pain, vomiting and overall complications following tonsillectomy in adults: a systematic review and meta-analysis of randomised controlled trials. Clin Otolaryngol 2011; 36: 531-42

16. Tiippana EM, Hamunen K, Kontinen VK, Kalso E. Do surgical patients benefit from perioperative gabapentin/pregabalin? A systematic review of efficacy and safety. Anesth Analg 2007; 104: 1545-56

17. Hwang SH, Park IJ, Cho YJ, Jeong YM, Kang JM. The efficacy of gabapentin/pregabalin in improving pain after tonsillectomy: a meta-analysis. Laryngoscope 2016; 126: $357-66$

18. Sanders JG, Dawes PJ. Gabapentin for perioperative analgesia in otorhinolaryngology-head and neck surgery: systematic review. Otolaryngol Head Neck Surg 2016; 155: 893-903 
19. Liberati A, Altman DG, Tetzlaff J, et al. The PRISMA statement for reporting systematic reviews and metaanalyses of studies that evaluate health care interventions: explanation and elaboration. J Clin Epidemiol 2009; 62: e1-34

20. Morrison A, Polisena J, Husereau D, et al. The effect of English-language restriction on systematic review-based meta-analyses: a systematic review of empirical studies. Int J Technol Assess Health Care 2012; 28: 138-44

21. Higgins JP, Altman DG, Gøtzsche PC, et al. Cochrane bias methods group; Cochrane statistical methods group. The Cochrane collaboration's tool for assessing risk of bias in randomised trials. BMJ 2011; 343: d5928

22. Moore RA, Straube S, Aldington D. Pain measures and cutoffs - no worse than mild pain as a simple, universal outcome. Anesthesia 2013; 68: 400-12

23. Atef A, Fawaz AA. Intravenous paracetamol is highly effective in pain treatment after tonsillectomy in adults. Eur Arch Otorhinolaryngol 2008; 265: 351-5

24. Salonen A, Silvola J, Kokki H. Does 1 or 2 g paracetamol added to ketoprofen enhance analgesia in adult tonsillectomy patients? Acta Anaesthesiol Scand 2009; 53: 1200-6

25. Parker DA, Gibbin KP, Noyelle RM. Syrup formulations for post-tonsillectomy analgesia: a double-blind study comparing ibuprofen, aspirin and placebo. J Laryngol Otol 1986; 100: 1055-60

26. Rorarius MG, Baer GA, Siirtola M, Lahti T, Laippala P. Effect of intravenous diclofenac or indomethacin on the emergence from anaesthesia for tonsillectomy. Acta Anaesthesiol Scand 1993; 37: 616-21

27. Tarkkila P, Saarnivaara L. Ketoprofen, diclofenac or ketorolac for pain after tonsillectomy in adults? $\mathrm{Br} J$ Anaesth 1999; 82: 56-60

28. Salonen A, Kokki H, Tuovinen K. IV ketoprofen for analgesia after tonsillectomy: comparison of pre- and postoperative administration. Br J Anaesth 2001; 86: 377-81

29. Naesh O, Niles LA, Gilbert JG, et al. A randomized, placebocontrolled study of rofecoxib with paracetamol in early post-tonsillectomy pain in adults. Eur J Anaesthesiol 2005; 22: $768-73$

30. Ismail SA, Mowafi HA. Preoperative peritonsillar lornoxicam infiltration is not superior to intravenous lornoxicam for pain relief following tonsillectomy in adults. Eur $\mathrm{J}$ Anaesthesiol 2010; 27: 807-11

31. Mowafi HA, Telmessani L, Ismail SA, Naguib MB. Preoperative lornoxicam for pain prevention after tonsillectomy in adults. J Clin Anesth 2011; 23: 97-101

32. Xie JR, Zhu YM, Zhang LF, Pang YJ, Yu LN. Effect of perioperative administration of parecoxib on post-tonsillectomy pain in adults. Afr J Pharm Pharmacol 2012; 6: 2141-7

33. Ng TT, Diamantaras D, Priestley J, Redman J, De Silva N, Mahanta V. Is celecoxib a useful adjunct in the treatment of post-tonsillectomy pain in the adult population? A randomised, double-blind, placebo-controlled study. J Laryngol Otol 2017; 131: S18-28

34. Fields M, Cabraal D, Dawes P. The effect of dexamethasone on post operative pain following tonsillectomy in adults. Aust J Otolaryngol 1994; 1: 426-9

35. Carr MM, Williams JG, Carmichael L, Nasser JG. Effect of steroids on posttonsillectomy pain in adults. Arch Otolaryngol Head Neck Surg 1999; 125: 1361-4

36. Stewart R, Bill R, Ullah R, McConaghy P, Hall SJ. Dexamethasone reduces pain after tonsillectomy in adults. Clin Otolaryngol Allied Sci 2002; 27: 321-6
37. Al-Shehri AM. Steroid therapy for post-tonsillectomy symptoms in adults: a randomized, placebo-controlled study. Ann Saudi Med 2004; 24: 365-7

38. Rujirojindakul P, Atchariyasathian V, Uakritdathikran T, Boonyata N, Saefung B, Jitmun P. Effect of dexamethasone on postoperative pain after adult tonsillectomy. Thai $J$ Anesthesiol 2008; 34: 1-8

39. Lachance M, Lacroix Y, Audet N, Savard P, Thuot F. The use of dexamethasone to reduce pain after tonsillectomy in adults: a double-blind prospective randomized trial. Laryngoscope 2008; 118: 232-6

40. Vaiman M, Aviram E, Krakovski D, Gavriel H, Eviatar E. Electromyographic assessment of dexamethasone in treatment of post-tonsillectomy pain: randomized, placebo-controlled trial. Am J Med Sci 2011; 341: 469-73

41. Vaiman M, Krakovski D, Haitov Z. Oxycodone and dexamethasone for pain management after tonsillectomy: a placebo-controlled EMG assessed clinical trial. Med Sci Monit 2011; 17: PI25-31

42. Thimmasettaiah NB, Chandrappa RG. A prospective study to compare the effects of pre, intra and post operative steroid (dexamethasone sodium phosphate) on post tonsillectomy morbidity. J Pharmacol Pharmacother 2012; 3: 254-8

43. Khafagy AH, Osman SM. Preoperative administration of dexamethasone reduces post-tonsillectomy morbidities in adults. Egypt J Ear Nose Throat Allied Sci 2013; 14: $113-7$

44. Mikkelsen S, Hilsted KL, Andersen PJ, et al. The effect of gabapentin on post-operative pain following tonsillectomy in adults. Acta Anaesthesiol Scand 2006; 50: 809-15

45. Jeon EJ, Park YS, Park SS, Lee SK, Kim DH. The effectiveness of gabapentin on post-tonsillectomy pain control. Eur Arch Otorhinolaryngol 2009; 266: 1605-9

46. Abdelmageed W, Abdelrazik S, Nassar A, Abdelkawi M. Analgesic effects of gabapentine in tonsillectomy. Egypt $J$ Hosp Med 2010; 38: 51-8

47. Mathiesen O, Jørgensen DG, Hilsted KL, et al. Pregabalin and dexamethasone improves post-operative pain treatment after tonsillectomy. Acta Anaesthesiol Scand 2011; 55: 297-305

48. Kawamata T, Omote K, Kawamata M, Namiki A. Premedication with oral dextromethorphan reduces postoperative pain after tonsillectomy. Anesth Analg 1998; 86: $594-7$

49. Rafiei MR, Aghadavoudi O, Rezvani M, Poorqasemian M. Evaluation of preemptive analgesia with dextromethorphan gargling in patients undergoing tonsillectomy. J Res Med Sci 2012; 17: S200-3

50. Van Elstraete AC, Lebrun T, Sandefo I, Polin B. Ketamine does not decrease postoperative pain after remifentanilbased anaesthesia for tonsillectomy in adults. Acta Anaesthesiol Scand 2004; 48: 756-60

51. Vaiman M, Krakovski D. EMG assessment of analgesia in treatment of posttonsillectomy pain. Clin J Pain 2012; 28: $143-8$

52. Barden J, Derry S, McQuay HJ, Moore RA. Single dose oral ketoprofen and dexketoprofen for acute postoperative pain in adults. Cochrane Database Syst Rev 2009; 7: CD007355

53. Dahl JB, Nielsen RV, Wetterslev J, et al. Post-operative analgesic effects of paracetamol, NSAIDs, glucocorticoids, gabapentinoids and their combinations: a topical review. Acta Anaesthesiol Scand 2014; 58: 1165-81 
54. Plante J, Turgeon AF, Zarychanski R, et al. Effect of systemic steroids on post-tonsillectomy bleeding and reinterventions: systematic review and meta-analysis of randomised controlled trials. BMJ 2012; 345: e5389

55. Suzuki S, Yasunaga H, Matsui H, Horiguchi H, Fushimi K, Yamasoba $\mathrm{T}$. Impact of systemic steroids on posttonsillectomy bleeding: analysis of 61430 patients using a national inpatient database in Japan. JAMA Otolaryngol Head Neck Surg 2014; 140: 906-10

56. Price DD, Mayer DJ, Mao J, Caruso FS. NMDA-receptor antagonists and opioid receptor interactions as related to analgesia and tolerance. J Pain Symptom Manage 2000; 19: S7-11
57. Duedahl TH, Rømsing J, Møiniche S, Dahl JB. A qualitative systematic review of peri-operative dextromethorphan in post-operative pain. Acta Anaesthesiol Scand 2006; 50: $1-13$

58. Bell RF1, Dahl JB, Moore RA, Kalso E. Peri-operative ketamine for acute post-operative pain: a quantitative and qualitative systematic review (Cochrane review). Acta Anaesthesiol Scand 2005; 49: 1405-28

59. Cho HK, Kim KW, Jeong YM, Lee HS, Lee YJ, Hwang SH. Efficacy of ketamine in improving pain after tonsillectomy in children: meta-analysis. PLoS One 2014; 9, e101259

Handling editor: J.G. Hardman 